\title{
Increased sedation requirements in patients receiving extracorporeal membrane oxygenation for respiratory and cardiorespiratory failure
}

\author{
K. SHEKAR*, J. A. ROBERTS $\dagger$, D. V. MULLANY $\$$, A. CORLEY§, S. FISQUET**, T. N. BULL††, \\ A. G. BARNETT杮, J. F. FRASER § \\ Critical Care Research Group, Adult Intensive Care Unit, The Prince Charles Hospital, Brisbane, Queensland, Australia
}

\begin{abstract}
SUMMARY
Critically ill patients receiving extracorporeal membrane oxygenation (ECMO) are often noted to have increased sedation requirements. However, data related to sedation in this complex group of patients is limited. The aim of our study was to characterise the sedation requirements in adult patients receiving ECMO for cardiorespiratory failure. A retrospective chart review was performed to collect sedation data for 30 consecutive patients who received venovenous or venoarterial ECMO between April 2009 and March 2011. To test for a difference in doses over time we used a regression model. The dose of midazolam received on ECMO support increased by an average of $18 \mathrm{mg}$ per day (95\% confidence interval $8,29 \mathrm{mg}, P=0.001$ ), while the dose of morphine increased by $29 \mathrm{mg}$ per day ( $95 \%$ confidence interval $4,53 \mathrm{mg}, P=0.021)$ The venovenous group received a daily midazolam dose that was $157 \mathrm{mg}$ higher than the venoarterial group (95\% confidence interval $53,261 \mathrm{mg}, P=0.005)$. We did not observe any significant increase in fentanyl doses over time (95\% confidence interval $1269,4337 \mu \mathrm{g}, P=0.94$ ). There is a significant increase in dose requirement for morphine and midazolam during ECMO. Patients on venovenous ECMO received higher sedative doses as compared to patients on venoarterial ECMO. Future research should focus on mechanisms behind these changes and also identify drugs that are most suitable for sedation during ECMO.
\end{abstract}

Key Words: extracorporeal membrane oxygenation, sedation and analgesia, drug toxicity, cardiorespiratory failure, pharmacokinetics

Extracorporeal membrane oxygenation (ECMO) temporarily supports patients with severe cardiac and/or respiratory failure that is not responsive to maximal conventional treatment ${ }^{1}$. It is usually a bridge to organ recovery, but can also be used as a bridge to long-term mechanical assist devices or transplantation ${ }^{2,3}$. Its role extends even further as an

* MB, BS, FCICM, Staff Specialist, Adult Intensive Care Unit, Critical Care Research Group and University of Queensland.

$\dagger$ BAppSc, BPharm, PhD, Senior Clinical Pharamcist, Royal Brisbane and Women's Hospital and Burns Trauma and Critical Care Research Centre, University of Queensland.

\$ MB, BS, FFICANZCA, Senior Staff Specialist, Adult Intensive Care Unit, Critical Care Research Group and University of Queensland.

$\S$ Nurse Researcher, Adult Intensive Care Unit; Critical Care Research Group and Griffith University.

**BPharm, Senior Clinical Pharmacist, Critical Care Research Group.

$\dagger$ Clinical Research Nurse, Adult Intenisve Care Unit and Critical Care Research Group.

$+\mathrm{PhD}$, Associate Professor, Institute of Health and Biomedical Innovation and School of Public Health, Queensland University of Technology and Critical Care Research Group.

$\S \S \mathrm{MB}, \mathrm{ChB}, \mathrm{PhD}, \mathrm{MRCP}, \mathrm{FRCA}$, FFARCSI, FCICM, Senior Staff Specialist, Adult Intensive Care Unit and Director, Critical Care Research Group.

Address for correspondence: Dr K. Shekar, The Prince Charles Hospital, Rode Road, Chermside, Qld 4032. Email: kiran_shekar@health.qld.gov.au

Accepted for publication on March 6, 2012. adjunct to cardiopulmonary resuscitation $(\mathrm{CPR})^{4}$, as well as high risk percutaneous cardiac interventions. Improvements in equipment, patient selection and a better understanding of extracorporeal circuit technology have resulted in some centres reporting significantly improved outcomes ${ }^{6-8}$. The Conventional Ventilation or ECMO for Severe Adult Respiratory Failure (CESAR) trial', and the Australian and New Zealand Intensive Care Society ECMO investigator study into Influenza A $(\mathrm{H} 1 \mathrm{~N} 1)^{10}$, have demonstrated its usefulness in the advanced management of respiratory failure unresponsive to maximal conventional therapy ${ }^{11-14}$. Data from the Extracorporeal Life Support Organ-ization registry ${ }^{15}$ suggest an increasing use of ECMO in adults with refractory respiratory and/or cardiac failure, with a reported survival of $63 \%$ for venovenous (VV) ECMO and 53\% for venoarterial (VA) ECMO.

ECMO is a highly invasive treatment in a group of patients who have failed conventional treatment and optimal sedation for this complex group of patients is not clearly defined. Sedative and analgesic medications promote patient 
comfort and eliminate pain, anxiety, agitation, delirium and other forms of distress induced by the intensive care unit (ICU) environment ${ }^{16}$. However, excessive sedation may lead to adverse outcomes. Use of objective scoring systems, sedation protocols ${ }^{17}$ allowing lighter sedation with daily interruptions $\mathbf{s}^{17-19}$ and minimising administration of paralytic agents have all been shown to reduce morbidity in ICU. Although evidence-based, these practices may not always be applicable to a critically ill patient on ECMO. Sedation during ECMO minimises the risk of catheter malposition or dislodgement as well as coughing, which induces 'suck down' or chatter, resulting in haemolysis. Deep sedation and paralysis may be required, especially early in the course of VV ECMO to achieve better circuit flows, minimise oxygen consumption and optimise ventilation. These factors may lead to a degree of 'permissiveness' when sedating patients during ECMO. To overcome this problem, some centres perform an early tracheostomy ${ }^{20}$ but the risks of this procedure in a patient on ECMO cannot be underestimated. Although neonatal studies have highlighted the increased sedation requirements during $\mathrm{ECMO}^{21-24}$, no such data is available for adult patients.

The aim of the study was to retrospectively review the sedative and analgesic drug requirements in adult patients receiving ECMO in our intensive care unit.

\section{METHODS}

\section{Study design and participants}

A retrospective chart review was performed after obtaining local ethics approval (HREC/11/ $\mathrm{QPCH} / 3)$. All patients undergoing ECMO since the commencement of the program at the Prince Charles Hospital, Brisbane, from April 2009 to February 2011 were included. One patient was excluded as death occurred in the operating room before meaningful data was charted.

\section{Details of sedation, ventilation and ECLS}

Our local protocol requires patients to be heavily sedated (equivalent to a Riker SedationAgitation Scale of 1 to 2) during the first few days of ECMO. This is particularly relevant for patients receiving VV ECMO to minimise oxygen consumption, optimise ECMO flows and ventilation. The senior nurses at the bedside titrate sedation and analgesia to patient comfort, degree of agitation, delirium and distress while preventing inadvertent disconnection of the circuit. Sedation is lightened where possible to assess neurology on a regular basis. Midazolam is used for sedation in all patients. Morphine is used for analgesia in patients with conserved renal function and fentanyl is used in patients with renal dysfunction or those requiring renal replacement therapy (RRT). Patients requiring additional sedation are given propofol, dexmedetomidine and thiopentone. Where necessary patients also may be prescribed enteral diazepam and/or neuroleptic agents if clinically indicated. Sedation is rapidly de-escalated after discontinuation of ECMO, particularly where a tracheostomy is performed.

The patients in the $\mathrm{VV}$ group receive a tidal volume of less than $6 \mathrm{ml} / \mathrm{kg}$ lean body weight with a positive end-expiratory pressure of 10 to $15 \mathrm{cmH}_{2} \mathrm{O}$, limiting plateau pressures and fractional inspired oxygen $\left(\mathrm{FiO}_{2}\right)$ to less than $30 \mathrm{cmH}_{2} \mathrm{O}$ and 0.5 respectively. Ventilator settings in the VA group were chosen to offer adequate lung aeration, prevention of atelectasis, avoidance of overdistension and prevention of coronary and cerebral hypoxemia (tidal volume 6 to $8 \mathrm{ml} / \mathrm{kg}$ lean body weight, rate 6 to 12 breaths/minute, $\mathrm{FiO}_{2} \leq 0.5$, positive end-expiratory pressure $>5 \mathrm{cmH}_{2} \mathrm{O}$, end-tidal carbon dioxide -20 to $30 \mathrm{mmHg}$ ).

The standard ECMO circuitry comprised polyvinyl chloride tubing, centrifugal pump (Jostra Rotaflow $^{\mathrm{TM}}$, Maquet, Germany) and a hollow fibre oxygenator (Quadrox $\mathrm{D}^{\mathrm{TM}}$, Maquet, Germany). RRT included both continuous (Aquarius ${ }^{\mathrm{TM}}$, Baxter International Inc., USA), using a percutaneous vascular catheter, and extended daily diafiltration (ARrT Plus ${ }^{\mathrm{TM}}$, Fresenius USA Inc., USA) directly via the ECMO circuit.

\section{Data collection}

Data collection from the medical record was performed using a detailed data collection instrument. Hourly total doses of midazolam, fentanyl and morphine including infusion and boluses were recorded from the time of ECMO commencement to the end of the day when ECMO was ceased. Drug dosing is described as total daily dose and was the study variable unless otherwise specified. Demographic and severity of illness data were obtained from the local ECMO database. Sequential Organ Failure Assessment (SOFA) scores were recorded on days 1,3 and 5 of the ICU stay, and Acute Physiology and Chronic Health Evaluation (APACHE) III scores within the first 24 hours of ICU stay.

\section{Statistical analysis}

To provide a visual display of drug dosing over time we plotted the daily drug dose for each patient. We also plotted the average drug dose per day to show the average dosing over time. To test for 
differences in drug dosing over time we used a regression model. To allow each patient to have a different profile over time we used a mixed effects model and fitted a random intercept and slope for each patient. We examined the residuals of the final model to check for normality. We also included variables for RRT and VA/VV ECMO to examine differences in these patients both on average and over time. To examine the association between APACHE III and SOFA scores and midazolam dose, we used scatter plots and linear regression. These analyses used each patient's mean midazolam dose over their entire ECMO run. Results are presented as means and 95\% confidence intervals. We used a two-sided statistical significance level of 0.05 . Analyses were performed using $\mathrm{R}$ version 2.12.2 (www.r-project.org).

\section{RESULTS}

Of the 29 patients analysed, 13 received VV and 16 received VA ECMO. In total, 342 days of ECMO support was cumulatively received by all patients; VV, median 356 hours (range 52 to 645 hours); VA, median 160 hours (range 0 to 514 hours). The patient characteristics and severity of illness data are presented in Table 1.

Patients received between 0 and $1440 \mathrm{mg}$ per day of midazolam. The median daily midazolam dose was $175 \mathrm{mg}$ (range 24 to $1092 \mathrm{mg}$ ). Figure 1 and Table 2 show the summary results for midazolam, morphine and fentanyl. On average, the daily dose of midazolam increased by $18 \mathrm{mg}$ per day $(95 \%$ confidence interval $8,29 \mathrm{mg}, P=0.001)$ after commencement of ECMO, representing an increased dose of $10.2 \%$. The VV group had a daily midazolam dose $157 \mathrm{mg}$ higher on average than the VA group (95\% confidence interval $53,261 \mathrm{mg}, P=0.005)$. There was no statistically significant difference in average dose of midazolam for the RRT group $(P=0.16)$. On average, the daily dose of morphine increased by $29 \mathrm{mg}$ per day (95\% confidence interval $4,53 \mathrm{mg}, P=0.02$ ). There was no statistically significant increase in fentanyl doses over time $(P=0.94)$. Fifteen of the 29 patients required propofol (100 to $300 \mathrm{mg} /$ hour) as a rescue agent at various times and there was an increased use between days 3 and 8 . Nine of these 15 patients received VV ECMO. Dexmedetomidine and thiopentone were used in two patients. All patients on VV ECMO received boluses of a neuromuscular blocker (vecuronium, median daily dose $20 \mathrm{mg}$, range 20 to $40 \mathrm{mg} /$ day) early in their course of ECMO.

In Figure 2 the predicted daily doses of midazolam, fentanyl and morphine are graphed according to whether RRT was used. There was no significant influence of RRT on average doses over time (one to 21 days) for midazolam, morphine and fentanyl in both VA and VV groups. In Figure 3, measures of sickness severity, SOFA and APACHE III scores are compared with

TABLE 1

Demographic and severity of illness data

\begin{tabular}{lll}
\hline & VV ECMO & VA ECMO \\
\hline Age, median, y & $35(18-55)$ & $54(16-77)$ \\
Gender, male (\%) & $6(46)$ & $12(71)$ \\
Admission weight, kg & $71.5(47-106)$ & $79(44-112)$ \\
Duration of ECMO, h & $356(52-645)$ & $160(1-514)$ \\
APACHE II score & $20(16-35)$ & $21(9-39)$ \\
APACHE III score & $80(51-123)$ & $79(48-128)$ \\
APACHE III ROD estimate & $0.39(0.13-0.73)$ & $0.36(0.04-0.91)$ \\
SOFA score day 1 & $10(6-16)$ & $10(6-16)$ \\
SOFA score day 3 & $10(5-17)$ & $10(4-17)$ \\
SOFA score day 5 & $11(4-14)$ & $10(5-11)$ \\
Pre-ECMO creatinine, $\mu \mathrm{mol} / 1$ & $166(35-422)$ & $127(65-383)$ \\
Pre-ECMO bilirubin, $\mu \mathrm{mol} / 1$ & $20(4-189)$ & $35(6-101)$ \\
PRBC transfusion, units & $11(4-53)$ & $31(8-191)$ \\
\hline
\end{tabular}

$\mathrm{VV}=$ venovenous, $\mathrm{ECMO}=$ extracorporeal membrane oxygenation, $\mathrm{VA}=$ venoarterial, APACHE $=$ Acute Physiology and Chronic Health Evaluation, ROD $=$ risk of death, SOFA $=$ Sequential Organ Failure Assessment, $\mathrm{PRBC}=$ packed red blood cells. 
daily midazolam dose. Higher SOFA scores were associated with lower doses, although this association was of borderline statistical significance $(P=0.053)$. Higher APACHE III scores were non-significantly associated with lower midazolam doses $(P=0.14)$. Severity of illness had no significant impact on the doses of morphine and fentanyl.

\section{DISCUSSION}

This study highlights the challenges in maintaining optimal sedation in patients receiving ECMO. The data presented are unique and advance our understanding of sedative drug use during ECMO, which is at present very limited. In general, most patients received increasing doses of midazolam

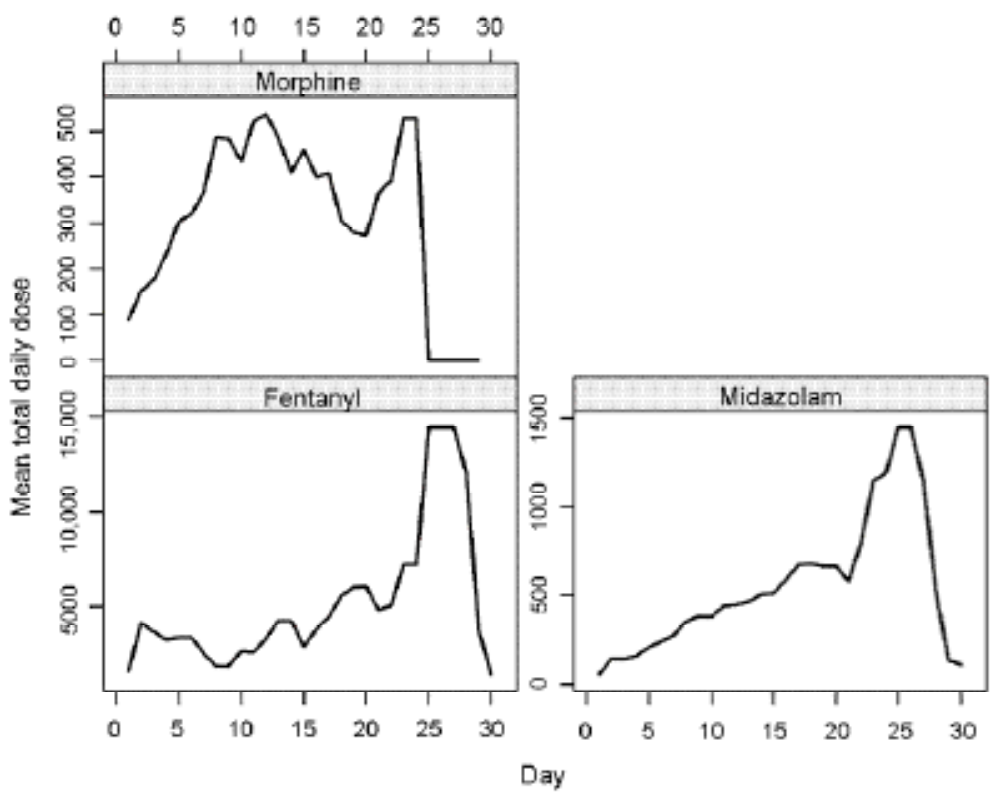

FIGURE 1: Average dose requirements for morphine, midazolam and fentanyl appear to increase with the duration of ECMO (Fentanyl doses in micrograms, morphine and midazolam doses in milligrams). In some patients, this occurred despite the concurrent use of other sedative agents (propofol, dexmedetomidine and thiopentone). Tracheostomy on liberation from ECMO led to a significant decrease in sedative and analgesic drug requirements seen between days 25 to 30 . This data is particularly influenced by patients on venovenous ECMO.

TABLE 2

Mixed model results for midazolam, morphine and fentanyl

\begin{tabular}{lllll}
\hline Drug & Variable & Mean & $95 \%$ CI & $P$ value \\
\hline Midazolam & Day & 18 & 8,29 & 0.001 \\
& RRT $=\mathrm{Y}$ & -72 & $-175,32$ & 0.164 \\
\multirow{5}{*}{ Morphine } & VA/VV=VV & 157 & 53,261 & 0.005 \\
& Day & 29 & 4,53 & 0.021 \\
& RRT $=\mathrm{Y}$ & -23 & $-247,202$ & 0.83 \\
\multirow{3}{*}{ Fentanyl } & VA/VV=VV & 129 & $-103,361$ & 0.24 \\
& Day & -7 & $-198,184$ & 0.94 \\
& RRT $=\mathrm{Y}$ & 419 & $-1426,2264$ & 0.64 \\
& VA/VV=VV & 166 & $-1698,2030$ & 0.86
\end{tabular}

To allow each patient to have a different profile over time we used a mixed effects model and fitted a random intercept and slope for each patient to examine changes in the daily doses of midazolam, morphine and fentanyl over time with or without RRT, and with or without VV or VA ECMO. On average the 24-hour dose increased by $18 \mathrm{mg}$ for midazolam and $29 \mathrm{mg}$ for morphine from the day of commencement of ECMO. There was no significant increase in fentanyl doses over time. $\mathrm{CI}=$ confidence interval, $\mathrm{RRT}=$ renal replacement therapy, $\mathrm{Y}=$ yes, $\mathrm{VA}=$ venoarterial, $\mathrm{VV}=$ venovenous. 
and morphine with time. This occurred despite the concurrent use of multiple sedative agents like propofol, dexmedetomidine, thiopentone and neuroleptic drugs in some patients. Interestingly, in this small group of patients RRT did not significantly influence the drug doses used. The difference in the severity of illness between patients receiving VA and VV ECMO was not significant enough to explain the higher dose requirement in the VV group. Patients in the VV group are more critically balanced from
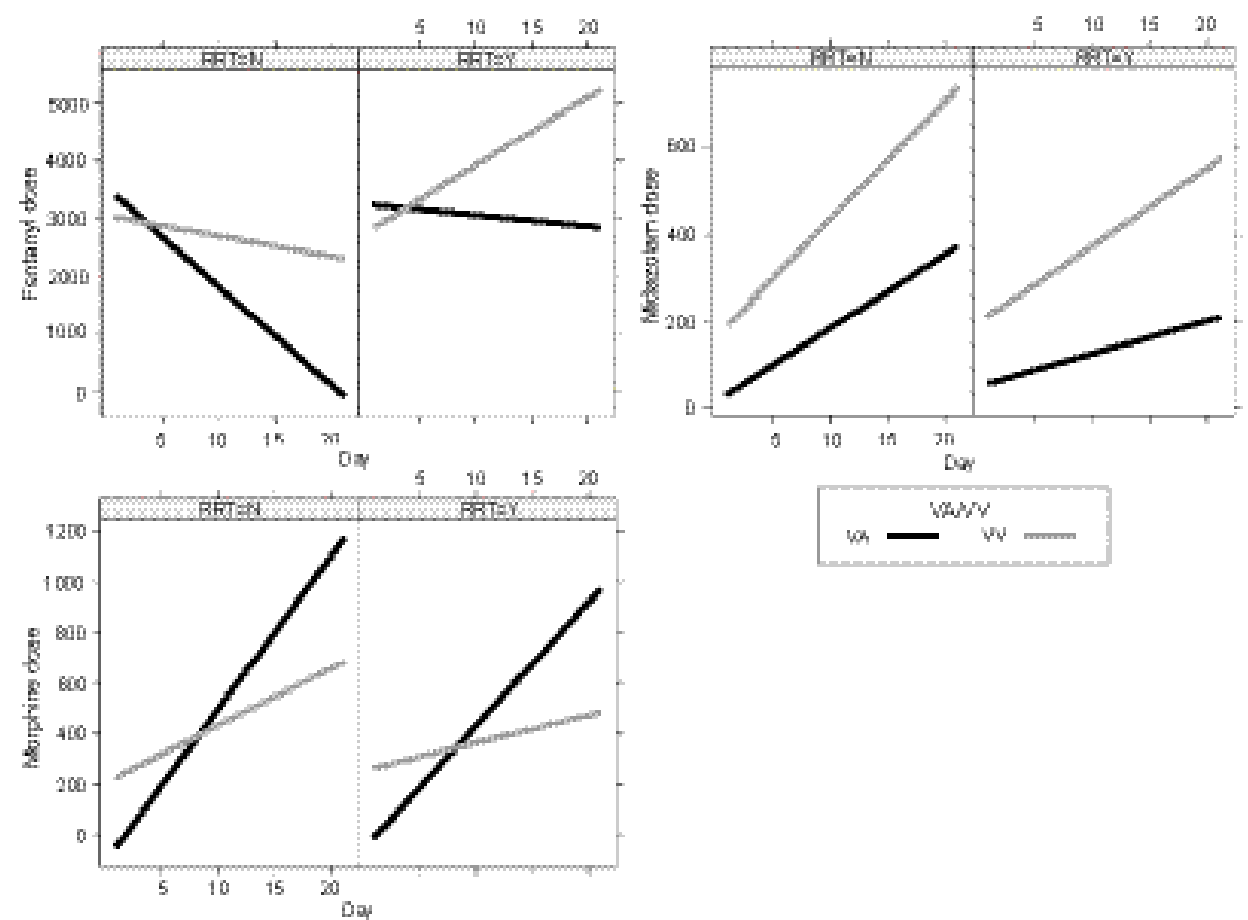

FIGURE 2: Average doses for all patients over time (one to 21 days) for midazolam, morphine (doses in milligrams) and fentanyl (doses in micrograms). Data is presented as to whether the patient was receiving renal replacement therapy (RRT) with the left panels representing doses without RRT and the panels on the right describing data in the presence of RRT. The grey line represents data from patients receiving venovenous ECMO and the black line represents data from patients receiving venoarterial $\mathrm{ECMO} . \mathrm{N}=$ no, $\mathrm{Y}=$ yes, $\mathrm{VA}=$ venoartertial, $\mathrm{VV}=$ venovenous.
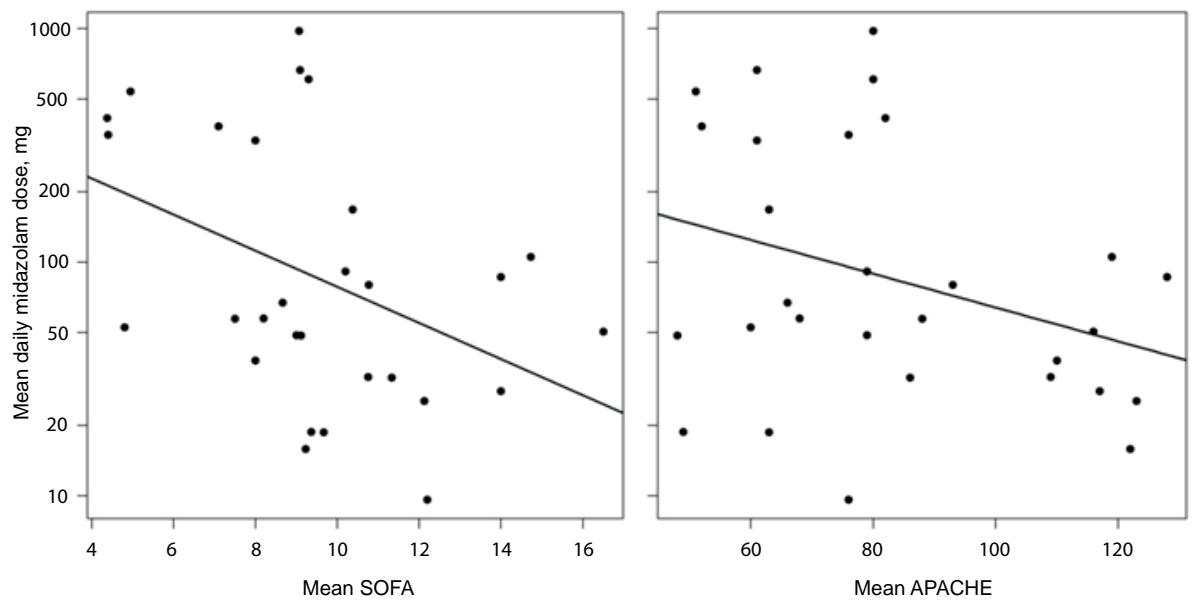

FIGURE 3: Severity of illness and midazolam requirement during ECMO. Mean 24-hourly midazolam dose for all patients plotted against the average Sequential Organ Failure Assessment (SOFA) score (left panel) and Acute Physiology and Chronic Health Evaluation (APACHE) II score (right panel). A log scale was used on the $\mathrm{Y}$ axis to account for two patients who received very high doses. The fitted line is from a regression with log-transformed dose as the dependent variable and the SOFA or APACHE score as the independent variable. The R-squared for SOFA and midazolam is $42 \%$ and the $P$ value is 0.024 . The R-squared for APACHE and midazolam is $33 \%$ and the $P$ value is 0.083 . 
an oxygenation point of view and are very dependent on optimal circuit flows, especially early in their course of ECMO. While this may explain the increased sedation requirements early in the course of VV ECMO, the mechanisms behind the escalating sedation needs later in the course of $\mathrm{VV}$ ECMO in patients with resolving lung injury are poorly understood.

It is unclear if the very high sedation requirement seen in our patients is entirely attributable to circuit factors alone. Mechanical ventilation, bleeding, delaying tracheostomy until liberation from ECMO, possible alterations in pharmacodynamics, development of tolerance and recovering organ function with time may all have contributed to the increase in sedation requirements. However, it is possible that ECMO may influence pharmacokinetics (PK) significantly and this effect may be more pronounced for certain drugs depending on their physicochemical properties. The common mechanisms are likely to include increased volume of distribution, decreased clearance and adsorption of the drugs in the circuit ${ }^{25}$. ECMO probably constitutes another compartment for drug distribution from a PK point of view. The pharmacodynamic effects of some of the sedative drugs may be further complicated by the presence of their active metabolites.

The ability of ECMO to alter the PK of sedative drugs has been highlighted in studies involving neonates and simulated ECMO circuits. Neonates receiving ECMO demonstrated an escalation of fentanyl and morphine doses with time ${ }^{26,27}$. Development of tolerance, organ maturation ${ }^{28}$ and resolution of organ failure may all account for increased drug clearance in the neonatal population in addition to circuit factors. Clearance of morphine and its metabolites was reduced in several studies and correlated with severity of illness ${ }^{29-31}$. Variable data exists regarding the $\mathrm{PK}$ of morphine and fentanyl in neonates receiving $\mathrm{ECMO}^{32,33}$.

Midazolam and its metabolites exhibited similar properties during ECMO in neonates. There were substantial increments in doses over time despite 1-hydroxy midazolam glucuronide accumulation during $\mathrm{ECMO}^{28,34,35}$. These effects have also been observed with lorazepam ${ }^{29,36}$. There is limited data on other sedative agents used during ECMO. A requirement for higher doses of phenobarbital has been reported ${ }^{22}$ during ECMO in neonates. The escalating sedation requirement with time in adult patients on ECMO appears to be similar to that in neonates despite the differences in development, physiology, PK and pharmacodynamics.
Simulated circuit studies using neonatal circuits and pumps confirm the potential role of the extracorporeal circuit in altering the PK of administered drugs ${ }^{2938}$. Though limited by variability in the technology used, these studies have clearly demonstrated the unpredictable behaviour of sedative drugs in the ECMO circuit ${ }^{21}$. Type of oxygenator $^{36}$, pump ${ }^{39}$ and age of the membrane ${ }^{40}$ have all been shown to have a variable influence on PK.

Limitations of currently available studies include the fact that some data are from ex vivo and in vitro models that do not account for the altered physiology common to critical illness. Other data from neonates may not be applicable to adults, due to the developmental and age-related physiological differences. The studies that do exist have used different equipment, such as polymethylpentene vs silicone membrane oxygenators, or uncoated tubing, which is different from presently available equipment. The mean duration of ECMO support has increased with the changing demographics of $\mathrm{ECMO}^{41}$ leading to the possibility of saturation of circuit binding sites over time ${ }^{42}$.

Critical illness and ECMO may have profound effects on the pharmacokinetics ${ }^{21,25,36,42-45}$ of drugs. Pathophysiological factors such as sickness severity, haemodilution ${ }^{42}$, altered serum protein concentrations $^{43}$, the inflammatory response induced by the extracorporeal circuit ${ }^{46}$, bleeding and massive transfusion, changes in the extracellular fluid volume and total body water may all lead to an increase in the volume of distribution of administered drugs. Drug adsorption and/or sequestration in the extracorporeal circuit tubing and the oxygenator may further increase the volume of distribution ${ }^{39}$. Organ dysfunction, changes in gastrointestinal ${ }^{43}$, renal, hepatic and pulmonary blood flow $^{47}$ as a result of a nonpulsatile circulation ${ }^{48,49}$ may result in decreased drug clearance. Renal replacement therapy can further complicate the PK of drugs by adding another parallel extracorporeal circuit that can potentially filter drugs ${ }^{50}$.

There are clear limitations to this study which include the absence of an ECMO-specific sedation scale and use of bispectral index monitoring ${ }^{51}$, the small sample size, absence of a control group and limited generalisability to other settings. Long-stay patients have more influence on the results over time due to their longer stays. The dose requirements for morphine, fentanyl and midazolam may have been much higher in the absence of other rescue drugs like propofol, thiopentone and dexmedetomidine, which 
were used in our patients. More detailed evaluation of organ function, total body water, extracellular fluid volume and the effects of RRT and its type are also required ${ }^{25}$. However, what is clear is that ECMO does dramatically increase sedative requirement and that this initial observational data supports the call for further prospective studies to improve safety and efficacy of ECMO.

The degree of variability seen in our study and limited existing data at present make it difficult to draw any meaningful conclusions on the use of sedative agents for patients on ECMO. However, the morbidity associated with excessive sedation calls for further research. Future studies should focus on development of sedation protocols that will allow optimal usage of available sedative drugs to meet the increased sedation requirements in this important subgroup of patients. It may be prudent to slowly taper opioid and benzodiazepine dosages after decannulation to prevent withdrawal. Our patients received regular enteral benzodiazepines and opioid replacement after decannulation to minimise risks of withdrawal.

\section{CONCLUSIONS}

This preliminary study highlights the sedationrelated issues in patients receiving ECMO. Unfortunately, a lack of knowledge of the underlying mechanisms presents challenges for clinicians. Future research should aim at characterising the effect of the circuit, drug and patient factors on the PK of sedatives and analgesics during ECMO. This may necessitate the development of ex vivo and large animal models of ECMO to better understand the circuit and host interations ${ }^{52}$. Identifying the sedative agents that are least affected by the PK alterations during $\mathrm{ECMO}^{53}$, development of ECMO specific sedation protocols and further refinements in ECMO circuitry may minimise morbidity associated with excessive sedation in this most unwell cohort of intensive care patients.

\section{ACKNOWLEDGEMENTS}

Dr Marc Ziegenfuss, Director, Adult Intensive Care services. Ms Amy Spooner and Ms Nicola Sharpe for their assistance during data acquisition.

\section{REFERENCES}

1. Bartlett RH, Gattinoni L. Current status of extracorporeal life support (ECMO) for cardiopulmonary failure. Minerva Anestesiol 2010; 76:534-540.

2. Strueber M. Bridges to lung transplantation. Curr Opin Organ Transplant 2011; 16:458-461.
3. Fitzgerald D, Ging A, Burton N, Desai S, Elliott T, Edwards L. The use of percutaneous ECMO support as a 'bridge to bridge' in heart failure patients: a case report. Perfusion 2010; 25:321$235,327$.

4. Thiagarajan RR, Brogan TV, Scheurer MA, Laussen PC, Rycus T, Bratton SL. Extracorporeal membrane oxygenation to support cardiopulmonary resuscitation in adults. Ann Thorac Surg 2009; 87:778-785.

5. Booth KL, Roth SJ, Perry SB, del Nido PJ, Wessel DL, Laussen PC. Cardiac catheterization of patients supported by extracorporeal membrane oxygenation. J Am Coll Cardiol 2002; 40:1681-1686.

6. Stulak JM, Dearani JA, Burkhart HM, Barnes RD, Scott PD, Schears GJ. ECMO cannulation controversies and complications. Semin Cardiothorac Vasc Anesth 2009; 13:176-182.

7. Riley JB, Scott PD, Schears GJ. Update on safety equipment for extracorporeal life support (ECLS) circuits. Semin Cardiothorac Vasc Anesth 2009; 13:138-145.

8. Bartlett RH, Gazzaniga AB, Jefferies MR, Huxtable RF, Haiduc NJ, Fong SW. Extracorporeal membrane oxygenation (ECMO) cardiopulmonary support in infancy. Trans Am Soc Artif Intern Organs 1976; 22:80-93.

9. Peek GJ, Mugford M, Tiruvoipati R, Wilson A, Allen E, Thalanany MM et al. Efficacy and economic assessment of conventional ventilatory support versus extracorporeal membrane oxygenation for severe adult respiratory failure (CESAR): a multicentre randomised controlled trial. Lancet 2009; 374:1351-1363.

10. Davies A, Jones D, Bailey M, Beca J, Bellomo R, Blackwell $\mathrm{N}$ et al. Extracorporeal Membrane Oxygenation for 2009 Influenza $\mathrm{A}(\mathrm{H} 1 \mathrm{~N} 1)$ Acute Respiratory Distress Syndrome. JAMA 2009; 302:1888-1895.

11. Lewandowski K. Extracorporeal membrane oxygenation for severe acute respiratory failure. Crit Care 2000; 4:156-168.

12. Pipeling MR, Fan E. Therapies for refractory hypoxemia in acute respiratory distress syndrome. JAMA 2010; 304:2521-2527.

13. Esan A, Hess DR, Raoof S, George L, Sessler CN. Severe hypoxemic respiratory failure: part 1 - ventilatory strategies. Chest 2010; 137:1203-1216.

14. Raoof S, Goulet K, Esan A, Hess DR, Sessler CN. Severe hypoxemic respiratory failure: part 2 - nonventilatory strategies. Chest 2010; 137:1437-1448.

15. ECLS Registry Report (International summary). Extracorporeal Life Support Organisation. Ann Arbor, MI: Extracorporeal Life Support Organisation, 2011.

16. Sessler CN, Wilhelm W. Analgesia and sedation in the intensive care unit:anoverview of the issues. CritCare2008;12(Suppl3):S1.

17. Brook AD, Ahrens TS, Schaiff R, Prentice D, Sherman G, Shannon $\mathrm{W}$ et al. Effect of a nursing-implemented sedation protocol on the duration of mechanical ventilation. Crit Care Med 1999; 27:2609-2615.

18. Kress JP, Vinayak AG, Levitt J, Schweickert WD, Gehlbach $\mathrm{BK}$, Zimmerman $\mathrm{F}$ et al. Daily sedative interruption in mechanically ventilated patients at risk for coronary artery disease. Crit Care Med 2007; 35:365-371.

19. Schweickert WD, Gehlbach BK, Pohlman AS, Hall JB, Kress JP. Daily interruption of sedative infusions and complications of critical illness in mechanically ventilated patients. Crit Care Med 2004; 32:1272-1276.

20. Linden V, Palmer K, Reinhard J, Westman R, Ehren H, Granholm $\mathrm{T}$ et al. High survival in adult patients with acute respiratory distress syndrome treated by extracorporeal membrane oxygenation, minimal sedation, and pressure supported ventilation. Intensive Care Med 2000; 26:1630-1637. 
21. Dagan O, Klein J, Gruenwald C, Bohn D, Barker G, Koren G. Preliminary studies of the effects of extracorporeal membrane oxygenator on the disposition of common pediatric drugs. Ther Drug Monit 1993; 15:263-266.

22. Elliott ES, Buck ML. Phenobarbital dosing and pharmacokinetics in a neonate receiving extracorporeal membrane oxygenation. Ann Pharmacother 1999; 33:419-422.

23. Rosen DA, Rosen KR. Midazolam for sedation in the paediatric intensive care unit. Intensive Care Med 1991; 17 (Suppl 1):S15-19.

24. Dagan O, Klein J, Bohn D, Koren G. Effects of extracorporeal membrane oxygenation on morphine pharmacokinetics in infants. Crit Care Med 1994; 22:1099-1101.

25. Buck ML. Pharmacokinetic changes during extracorporeal membrane oxygenation: implications for drug therapy of neonates. Clin Pharmacokinet 2003; 42:403-417.

26. Caron E, Maguire DP. Current management of pain, sedation, and narcotic physical dependency of the infant on ECMO. J Perinat Neonatal Nurs 1990; 4:63-74.

27. Arnold JH, Truog RD, Orav EJ, Scavone JM, Hershenson MB. Tolerance and dependence in neonates sedated with fentanyl during extracorporeal membrane oxygenation. Anesthesiology 1990; 73:1136-1140.

28. Ahsman MJ, Hanekamp M, Wildschut ED, Tibboel D, Mathot RAA. Population pharmacokinetics of midazolam and its metabolites during venoarterial extracorporeal membrane oxygenation in neonates. Clin Pharmacokinet 2010; 49:407-419.

29. Mulla H, Lawson G, von Anrep C, Burke MD, Upton DU, Firmin RK et al. In vitro evaluation of sedative drug losses during extracorporeal membrane oxygenation. Perfusion 2000; 15:21-26.

30. Peters JWB, Anderson BJ, Simons SHP, Uges DRA, Tibboel D. Morphine pharmacokinetics during venoarterial extracorporeal membrane oxygenation in neonates. Intensive Care Med 2005; 31:257-263.

31. Peters JWB, Anderson BJ, Simons SHP, Uges DRA, Tibboel D. Morphine metabolite pharmacokinetics during venoarterial extra corporeal membrane oxygenation in neonates. Clin Pharmacokinet 2006; 45:705-714.

32. Geiduschek JM, Lynn AM, Bratton SL, Sanders JC, Levy $\mathrm{FH}$, Haberkern CM et al. Morphine pharmacokinetics during continuous infusion of morphine sulfate for infants receiving extracorporeal membrane oxygenation. Crit Care Med 1997; 25:360-364.

33. Leuschen MP, Willett LD, Hoie EB, Bolam DL, Bussey ME, Goodrich PD et al. Plasma fentanyl levels in infants undergoing extracorporeal membrane oxygenation. J Thorac Cardiovasc Surg 1993; 105:885-891.

34. Mulla H, McCormack P, Lawson G, Firmin RK, Upton DR. Pharmacokinetics of midazolam in neonates undergoing extracorporeal membrane oxygenation. Anesthesiology 2003; 99:275-282.

35. Mulla H, Lawson G, Peek GJ, Firmin RK, Upton DR. Plasma concentrations of midazolam in neonates receiving extracorporeal membrane oxygenation. ASAIO J 2003; 49:41-47.

36. Bhatt-Meht V, Annich G. Sedative clearance during extracorporeal membrane oxygenation. Perfusion 2005; 20:309315.
37. Hammaren E, Rosenberg PH, Hynynen M. Coating of extracorporeal circuit with heparin does not prevent sequestration of propofol in vitro. Br J Anaesth 1999; 82:38-40.

38. Mehta NM, Halwick DR, Dodson BL, Thompson JE, Arnold JH. Potential drug sequestration during extracorporeal membrane oxygenation: results from an ex vivo experiment. Intensive Care Med 2007; 33:1018-1024.

39. Wildschut ED, Ahsman MJ, Allegaert K, Mathot RAA, Tibboel D. Determinants of drug absorption in different ECMO circuits. Intensive Care Med 2010; 36:2109-2116.

40. Rosen DA, Rosen KR, Silvasi DL. In vitro variability in fentanyl absorption by different membrane oxygenators. J Cardiothorac Anesth 1990; 4:332-335.

41. Roy BJ, Rycus P, Conrad SA, Clark RH. The changing demographics of neonatal extracorporeal membrane oxygenation patients reported to the Extracorporeal Life Support Organization (ELSO) Registry. Pediatrics 2000; 106:1334-1338.

42. Mulla HGL, Firmin RK, David RU. Drug isposition during extracorporeal membrane oxygenation. Paediatric and Perinatal Drug Therapy 2001; 4:109-120.

43. Boucher BA, Wood GC, Swanson JM. Pharmacokinetic changes in critical illness. Crit Care Clin 2006; 22:255-271, vi.

44. Varghese JM, Roberts JA, Lipman J. Pharmacokinetics and pharmacodynamics in critically ill patients. Curr Opin Anaesthesiol 2010; 23:472-478.

45. Roberts JA. Using PK/PD to optimize antibiotic dosing for critically ill patients. Curr Pharm Biotechnol 2011; 12:2070-2079.

46. McILwain RB, Timpa JG, Kurundkar AR, Holt DW, Kelly DR, Hartman YE et al. Plasma concentrations of inflammatory cytokines rise rapidly during ECMO-related SIRS due to the release of preformed stores in the intestine. Lab Invest 2010; 90:128-139.

47. Vardi A, Jessen ME, Chao RY, Brink LW, Levin DL, Johnson RL. Effect of extracorporeal membrane oxygenation flow on pulmonary capillary blood flow. Crit Care Med 1995; 23:726-732.

48. Many M, Soroff HS, Birtwell WC, Giron F, Wise H, Deterling RA Jr. The physiologic role of pulsatile and nonpulsatile blood flow. II. Effects on renal function. Arch Surg 1967; 95:762-767.

49. Shevde K, DeBois WJ. Pro: pulsatile flow is preferable to nonpulsatile flow during cardiopulmonary bypass. J Cardiothorac Anesth 1987; 1:165-168.

50. Schetz M. Drug dosing in continuous renal replacement therapy: general rules. Curr Opin Crit Care 2007; 13:645-651.

51. LeBlanc JM, Dasta JF, Kane-Gill SL. Role of the bispectral index in sedation monitoring in the ICU. Ann Pharmacother 2006; 40:490-500.

52. Shekar K, Fung YL, Diab S, Mullandy DV, McDonald CI, Dunster KR, et al. Development of simulated and ovine models of ECLS to improve understanding of circuit-host interactions. Critical Care and Resuscitation 2012; 14:105-111.

53. Shekar JA. Pharmacokinetic changes in patients receiving extracorporeal membrane oxygenation. J Crit Care 2012; 18 Apr [Epub ahead of print]. 